\title{
CONSISTENT THEORY OF ALPHA-DECAY
}

\begin{abstract}
In the framework of the Goldberger-Watson decay theory, we consider the $\alpha$-decay of nuclei as a transition between the initial bound state of the nucleus and scattering states of the continuum spectrum with $\alpha$-particle. The scattering wave functions for the $\alpha$-decay with arbitrary orbital angular momentum are derived in the quasiclassical approximation. The $\alpha$-particle is described by the square-integrable wave packet formed by these functions, whose amplitude exponentially grows outside the nucleus up to the wave front. The Moshinsky's distortions of the $\alpha$-wave front are analyzed. The derived general expression for the decay rate is approximated by the quasiclassical formula.

Keywords: decay theory, alpha-decay, tunneling, scattering theory.
\end{abstract}

\section{Introduction}

Among different kinds of nuclear decays, the most attention is paid for many years to $\alpha$-decay. The contemporary theory of $\alpha$-decay [1-24] is based mainly on the ideas of Gamov [25], as well as of Condon and Gurney [26]. It is always supposed that, at the initial moment $t=0$, there is a ready $\alpha$ particle bound inside the potential well, which approximates the nuclear interaction. The subsequent tunneling through the Coulomb barrier, surrounding the potential well, ensures the leaking of $\alpha$-particle from the nucleus. Such a situation is described by the Schrödinger equation having the same form as the equations for stationary bound levels $[3,5]$. The necessity to provide the decay of the nucleus forced Gamov and his followers to introduce the complex energy $E_{0}-i \Gamma / 2$ instead of the real energy of the level $E_{0}$, where $\Gamma$ stands for the width of the level. The corresponding wave vector of the emitted $\alpha$-particle is also complex: $\kappa=\kappa^{\prime}+i \kappa^{\prime \prime}$ with $\kappa^{\prime}>0, \kappa^{\prime \prime}<0$. As a consequence, the wave function, which describes the relative motion of nuclear fragments, diverges with the growing distance $r$ between them becoming not square-integrable.

Furthermore, it is always demanded that the energy levels of such quasibound states are determined by the quasiclassical Bohr-Sommerfeld quantization rule. In particular, trying to ensure this restriction for every direction inside the deformed nuclei, Ismail et al. [14] imposed a rather strange requirement that

(C) A.YA. DZYUBLIK, 2021

ISSN 2071-0194. Ukr. J. Phys. 2021. Vol. 66, No. 5 the depth of the nuclear potential well depends on the direction of the $\alpha$-particle emission. It is worth to recall that just the Bohr-Sommerfeld condition indicates the position of resonances in the case of the scattering of particles in the potential field (see, e.g., Ref. [27]). For $\alpha$-particles, the features of such a resonant scattering have been analyzed by Karpeshin et al. [28].

Most consistently, the time-dependent task of decay has been reduced to the stationary Schrödinger equation by Silisteanu et al. [10], who introduced the non-Hermitian Hamiltonian, which has complex eigenvalues $E$ and diverging eigenfunctions.

Sitenko [27] indicated that this divergence can be overcome, if the particle is described by the wave packet $\Psi(r, 0)$, formed by the scattering wave functions $\psi_{E}(r, t)$ with different real energies $E$ in a vicinity of the resonant energy $E_{0}$. He assumed that the wave packet should be localized at $t=0$ inside the nucleus in the region $0<r<R$. Then it begins to spread, leaking from the nucleus. The obtained wave function $\Psi(r, t)$ again exponentially grows with $r$, but has the sharp wave front at the point $r_{f}=v t$, where $v$ is the velocity of the $\alpha$-particle. This provides the normalization of $\Psi(r, t)$ to unity. In such an approach, the initial state $\Psi(r, 0)$ belongs to the continuum spectrum. It describes a ready $\alpha$-particle and the daughter nucleus. But a more adequate representation of $\Psi(r, 0)$ is given by the shell model, which considers all the nucleons as free particles contained in a potential well. In this case, all the occupied levels are 
lower than the continuum spectrum, and, therefore, this state of the parent nucleus occurs to be exactly bound. The wave functions of these bound states $\varphi_{a}$ are eigenfunctions of the unperturbed Hamiltonian $H_{0}$. Their coupling to the states of the continuum spectrum $\varphi_{b}$ with a ready $\alpha$-particle is realized by a residual interaction $V^{\prime}$, being the difference of the complete Hamiltonian $H$ and $H_{0}$. The transition from $\varphi_{a}$ to $\varphi_{b}$ is accompanied by the transfer of some energy of the intrinsic motion of nucleons in fragments into the energy of their relative motion $E$. The value of $E$ is real and lies already in the continuum spectrum.

To the best of my knowledge, there is the only paper by Silisteanu et al. [10], who employed the same idea, i.e., they considered a transition from the bound state into scattering states triggered by $V^{\prime}$. However, from the very beginning, the authors operated with complex energies and did not touch the space-time evolution of the wave function for the $\alpha$-decay.

It is necessary to mention also the papers by Gurvitz et al. [31-33], who developed the two-potential model of decay. They assumed that, at $t<0$, there was a bound level with the energy $E_{0}$ in a spherically symmetric potential $U(r)$, which includes the potential well at $0 \leq r \leq R$ and the impenetrable barrier of a constant height $U(r)=V_{0}$ at $R \leq r<\infty$, for which $V_{0}>E_{0}$. Then at $t=0$, another potential $W(r)$ is abruptly switched on, which transforms the initial potential $U(r)$ to a more realistic $V(r)=U(r)+W(r)$, including already the barrier of finite width. The authors considered the $W(r)$ as a perturbation, which ensures the decay of the initially bound state, and described the time evolution of the wave function by equations of the Goldberger-Watson decay theory [32]. The continuous spectrum in Refs. [29, 30] begins at the brim of the barrier $V_{0}$ and, therefore, does not overlap with the bound level $E_{0}$. But in this case, the energy conservation law forbids the decay of such a bound state. In order to overcome this difficulty and to remove some singularities, the authors introduced one more potential $\tilde{W}(r)=W(r)+V_{0}$. Such two-potential model was applied to the $\alpha$-decay in Refs. [21, 22].

In this paper, I consider the decay of the bound state $\varphi_{a}$ of the parent nucleus described by some version of the shell model without any sets of nucleons tightly bound into clusters. The clusters only appear due to a residual interaction $V^{\prime}$ between the nucleons. Such description lays in the framework of the Goldberger-Watson strict decay theory [32], who split the Hamiltonian $H$ into the unperturbed Hamiltonian $H_{0}$ and the perturbation $V^{\prime}$,

$H=H_{0}+V^{\prime}$.

For the separation of $H_{0}$ and $V^{\prime}$ from $H$, we can use Feshbach's projection operator formalism [10,33]. Note also that the Goldberger-Watson decay theory has been previously used by Jackson [18] for the calculation of the $\alpha$-decay width. However, he did not derive a square-integrable wave function, which would describe the $\alpha$-decay. This task is solved here.

The eigenfunctions $\varphi_{a}$ of the operator $H_{0}$ describe bound levels of the parent nucleus, while the eigenfunctions $\varphi_{b}^{+}$describe the states belonging to the continuous spectrum. The bound levels are drowned into this continuous spectrum. Therefore, the interaction $V^{\prime}$ coupling the states $\varphi_{a}$ and $\varphi_{b}^{+}$, leads to the decay of the initial state $\varphi_{a}$ of the parent nucleus. Note also that all the functions $\varphi_{a}$ and $\varphi_{b}^{+}$are orthogonal, because $H_{0}$ is a Hermitian operator. They form a complete set of basis vectors.

Applying such an approach, I consider the $\alpha$-decay as a transition between the initial bound state $\varphi_{a}$ of the parent nucleus and the final states in the continuous spectrum $\varphi_{b}^{+}$. In the inverse process of the $\alpha$ scattering, the level $\varphi_{a}$ manifests itself as a resonant compound state, where the energy of the captured $\alpha$-particle is shared among all the nucleons. In other words, in the state $\varphi_{a}$, the $\alpha$-particle is dissolved in the nucleus. In the initial moment $t=0$, there is only the parent nucleus described by the wave function $\Psi(0)=\varphi_{a}$ without any $\alpha$ particle. Afterward, the wave function of the nucleus $\Psi(t)$ attributes components $\sim \varphi_{b}^{+}$, whereas the amplitude of the state $\varphi_{a}$ exponentially attenuates. We shall see that a small value of the transmission coefficient $e^{-2 S}$ is only realized far from the Bohr-Sommerfeld restriction imposed on the energy of the decaying nucleus.

\section{Main Definitions}

Let the charge number of the parent nucleus be $Z$, and let the mass number be $A$. The nuclear decay is considered in the c.m. frame. Both the nuclear potential $V_{n}(\beta ; r)$ and the Coulomb one $V_{C}(\beta ; r)$, depending on the deformation parameter $\beta$, can be expanded in the series in $\beta$. The zeroth order terms 
$V_{n}(r)$ and $V_{C}(r)$ represent spherically symmetric potentials. The corrections to these potentials, dependent on $\beta$, can be accounted by means of the coupledchannels formalism [34]. Here, I only deal with the spherical case.

In the $\alpha$-decay channel, we have a ready $\alpha$-particle and the daughter nucleus, whose relative motion is determined by the radius-vector $\mathbf{r}$, while their intrinsic motion by the coordinates $\xi_{\alpha}$ and $\xi_{d}$, respectively. The same variables are used in the cluster model (see, e.g., [35]) for any separated group of two protons and two neutrons, as well as $A-4$ nucleons of the parent nucleus. The parent nucleus in the initial state, formed at $t=0$, contains no ready $\alpha$ particle. On the contrary, all the nucleons are initially free particles, moving in some central potential well.

Let, at $t=0$, this initial state be described by the function $\varphi_{a}=g_{p}(\xi, \mathbf{r})$, where $\xi=\left\{\xi_{\alpha}, \xi_{d}\right\}$, while the corresponding eigenvalue of $H_{0}$ be $\varepsilon_{a}=\mathcal{M}_{p} c^{2}+\mathcal{E}_{p}$, where $\mathcal{E}_{p}$ is the energy of the excited nuclear level, the subscript $p$ specifies the spin $I_{p}$, its projection $M_{p}$ on the quantization axis and any other quantum numbers. Hereafter, $\mathcal{M}_{p(d)}$ and $\mathcal{M}_{\alpha}$ are the masses, respectively, of the parent (daughter) nucleus and the $\alpha$-particle being in the ground state.

In the $\alpha$-decay channel, the unperturbed Hamiltonian $H_{0}$ is a sum of the kinetic energy operator of the relative motion of the fragments $K$, their potential energy $V(r)$, Hamiltonians for the internal motion of the daughter nucleus $H_{\text {in }}^{(d)}$ and the $\alpha$-particle $H_{\text {in }}^{(\alpha)}$ :

$H_{0}=K+V(r)+H_{\mathrm{in}}^{(\alpha)}+H_{\mathrm{in}}^{(d)}, \quad K=-\frac{\hbar^{2}}{2 \mu} \Delta_{\mathbf{r}}$,

where the reduced mass $\mu=\mathcal{M}_{d} \mathcal{M}_{\alpha} /\left(\mathcal{M}_{d}+\mathcal{M}_{\alpha}\right)$.

The eigenfunctions of $H_{0}$ are

$\varphi_{b}^{+}(\xi, \mathbf{r})=\psi_{\boldsymbol{\kappa}}^{+}(\mathbf{r}) g_{d}\left(\xi_{d}\right) g_{\alpha}\left(\xi_{\alpha}\right)$.

These wave functions must be still antisymmetrized $[3,10]$. The factors $g_{d}\left(\xi_{d}\right)$ and $g_{\alpha}\left(\xi_{\alpha}\right)$ describe the internal motion of clusters and are determined by the equations

$$
\begin{aligned}
& H_{\mathrm{in}}^{(\alpha)} g_{\alpha}\left(\xi_{\alpha}\right)=\mathcal{M}_{\alpha} c^{2} g_{\alpha}\left(\xi_{\alpha}\right), \\
& H_{\mathrm{in}}^{(d)} g_{d}\left(\xi_{d}\right)=\left(\mathcal{M}_{d} c^{2}+\mathcal{E}_{d}\right) g_{d}\left(\xi_{d}\right),
\end{aligned}
$$

where $\mathcal{E}_{d}$ is the excitation energy of the daughter nucleus, the subscript $d$ of $g_{d}\left(\xi_{d}\right)$ includes spin $I_{d}$, its projection $M_{d}$, etc.
The function $\psi_{\boldsymbol{\kappa}}^{+}(\mathbf{r})$, responsible for the relative motion of fragments, satisfies the Schrödinger equation

$\left[\frac{\hbar^{2}}{2 \mu} \Delta_{\mathbf{r}}-V(r)+E\right] \psi_{\boldsymbol{\kappa}}^{+}(\mathbf{r})=0$,

where $E=\hbar^{2} \kappa^{2} / 2 \mu$ is the energy of the relative motion of fragments.

The unperturbed energies associated with $\varphi_{b}^{+}(\xi, \mathbf{r})$ are

$\varepsilon_{b}=\left(\mathcal{M}_{d}+\mathcal{M}_{\alpha}\right) c^{2}+\mathcal{E}_{d}+E$.

The Coulomb field for bare uniformly charged nuclei is given by

$V_{\mathrm{C}}(r)_{\mathrm{b}}=\left\{\begin{array}{l}\frac{(Z-2) e^{2}}{R}\left[3-\frac{r^{2}}{R^{2}}\right], \quad 0 \leq r<R, \\ \frac{2(Z-2) e^{2}}{r}, \quad r>R,\end{array}\right.$

where $R$ is the nucleus radius.

Further, I shall only consider the decay of nuclei surrounded by electrons. In this case, the $\alpha$-particle moves in the field

$V(r)=V_{n}(r)+V_{\mathrm{C}}(r)$,

where $V_{n}(r)$ stands for the nuclear potential well and $V_{\mathrm{C}}(r)$ for the effective Coulomb field. At small distances, when the $\alpha$-particle moves inside the nucleus or under the barrier, the Coulomb contribution to within a small correction $\sim r^{2}$ is $[36,37]$

$V_{\mathrm{C}}(r) \approx V_{\mathrm{C}}(r)_{\mathrm{b}}-\Delta Q$,

where $\Delta Q$ is the energy transferred to electrons. In nonmetallic targets $\Delta Q=B_{p}-B_{d}$, where $B_{p}$ and $B_{d}$ are the electron binding energies of the parent and daughter atoms. The conductivity electrons give a small correction [37].

Respectively, the nuclear energies are related by $\varepsilon_{b} \approx \varepsilon_{a}-\Delta Q$ with uncertainty of the order of the decay width $\Gamma$. The relative energy $E$ of clusters in the $d$ th channel is spread about the mean energy $E_{d}$. For the decay by screened nuclei $[36,37]$,

$E_{d}=Q_{d}-\Delta Q$

where

$Q_{d}=\left(\mathcal{M}_{p}-\mathcal{M}_{\alpha}-\mathcal{M}_{d}\right) c^{2}+\mathcal{E}_{p}-\mathcal{E}_{d}$,

is the average nuclear energy released in this decay. 


\section{Scattering Wave Functions}

The functions $\psi_{\boldsymbol{\kappa}}^{+}(\mathbf{r})$ are normalized by

$\left\langle\psi_{\boldsymbol{\kappa}^{\prime}}^{+}(\mathbf{r}) \mid \psi_{\boldsymbol{\kappa}}^{+}(\mathbf{r})\right\rangle=\delta\left(\boldsymbol{\kappa}^{\prime}-\boldsymbol{\kappa}\right)$.

In the asymptotic region, $r \rightarrow \infty$, they are represented by a sum of the incident wave $(2 \pi)^{-3 / 2} e^{i \kappa \mathbf{r}}$ and a spherical outgoing wave $\sim \frac{1}{r} e^{i \kappa r}$.

The $\psi_{\kappa}^{+}(\mathbf{r})$ can be expanded in partial waves [32]:

$\psi_{\boldsymbol{\kappa}}^{+}(\mathbf{r})=\sum_{l=0}^{\infty} \sum_{m=-l}^{l} i^{l} e^{i \delta_{l}(\kappa)} \frac{w_{l}(\kappa ; r)}{\kappa r} Y_{l m}^{*}(\hat{\boldsymbol{\kappa}}) Y_{l m}(\hat{\mathbf{r}})$,

where $\hat{\boldsymbol{\kappa}}$ and $\hat{\mathbf{r}}$ denote the spherical angles of the vectors $\boldsymbol{\kappa}$ and $\mathbf{r}$, respectively. Here, the radial functions $w_{l}(\kappa ; r)$ satisfy the equation

$w_{l}^{\prime \prime}(\kappa ; r)-\left[l(l+1) / r^{2}+v(r)-\kappa^{2}\right] w_{l}(\kappa ; r)=0$,

where the reduced potential

$v(r)=2 \mu V(r) / \hbar^{2}$.

The regular functions at $r \rightarrow 0$ behave themselves

$w_{l}(\kappa ; r) \sim(\kappa r)^{l+1}$

With growing $r$, the screened Coulomb potential $V_{\mathrm{C}}(r)$ attenuates faster than a pure Coulomb one. Respectively, as $r \rightarrow \infty$, the functions $w_{l}(\kappa ; r)$ have a simpler asymptotics than the Coulomb functions [32]

$w_{l}(\kappa ; r) \approx \sqrt{\frac{2}{\pi}} \sin \left(\kappa r-\frac{l \pi}{2}+\delta_{l}(\kappa)\right)$,

where $\delta_{l}(\kappa)$ stands for the phase shift. These functions are normalized as follows:

$\int_{0}^{\infty} w_{l}(\kappa ; r) w_{l}\left(\kappa^{\prime} ; r\right) d r=\delta\left(\kappa-\kappa^{\prime}\right)$.

Since the Hamiltonian of the closed nuclear system $H$ is invariant with respect to rotations, it is more appropriate to expand the basis functions $\varphi_{b}^{+}(\xi, \mathbf{r})$ in terms of the eigenfunctions of the operators $\mathbf{I}^{2}$ and $I_{z}$, where $\mathbf{I}=\mathbf{I}_{d}+\mathbf{l}$ is the total angular momentum operator of the nuclear clusters, and $I_{z}$ is its projection on the quantization axis $z$. Due to such symmetry of $H$, the interaction $V$ couples the states with the same total spin and its projection, i.e., $I=I_{p}$ and $M=M_{p}$.

The spin of the daughter nucleus and the orbital angular momentum $\mathbf{l}$ are coupled, giving the eigenfunctions of $\mathbf{I}^{2}$ and $I_{z}$ :

$\mathscr{Y}_{I l I_{d}}^{M}(\xi, \hat{\mathbf{r}})=\sum_{m M_{d}}\left(l I_{d} m M_{d} \mid I M\right) Y_{l m}(\hat{\mathbf{r}}) g(\xi)$

where $g(\xi)=g_{d}\left(\xi_{d}\right) g_{\alpha}\left(\xi_{\alpha}\right)$ and $\left(j_{1} j_{2} m_{1} m_{2} \mid j m\right)$ are the Clebsch-Gordan coefficients. The inverse transformation is

$Y_{l m}(\hat{\mathbf{r}}) g(\xi)=\sum_{I M}\left(l I_{d} m M_{d} \mid I M\right) \mathscr{Y}_{I l I_{d}}^{M}(\xi ; \hat{\mathbf{r}})$.

Note that the functions $\mathscr{Y}_{I l I_{d}}^{M}(\xi ; \hat{\mathbf{r}})$ are analogs of the generalized spherical harmonics used by Newton [38]. By inserting (13) into (3) and using (20), I rewrite the wave function $\varphi_{b}^{+}$as

$\varphi_{b}^{+}(\xi, \mathbf{r})=\sum_{I M} \sum_{l=0}^{\infty} \frac{w_{l}(\kappa ; r)}{\kappa r} \times$

$\times \mathscr{Y}_{I l I_{d}}^{M}(\xi, \hat{\mathbf{r}}) \mathfrak{Y}_{I}^{M^{*}}\left(l I_{d} M_{d} ; \hat{\boldsymbol{\kappa}}\right)$,

where I introduced the notation

$\mathfrak{Y}_{I}^{M}\left(l I_{d} M_{d} ; \hat{\boldsymbol{\kappa}}\right)=$

$=i^{-l} e^{-i \delta_{l}} \sum_{m=-l}^{l}\left(l I_{d} m M_{d} \mid I M\right) Y_{l m}(\hat{\boldsymbol{\kappa}})$.

\section{Quasiclassical Approximation}

Let us solve the radial equation (14) in the quasiclassical approximation (WKB), making use of standard matching rules [39] (more refined matching has been applied recently in Ref. [40]). The WKB does not "work" at $r \approx 0$, when the effective potential quickly changes at the distance of the order of the wavelength [39]. In order to overcome this obstacle, Langer [41] replaced the variable $r$ by $x$ :

$r=\kappa^{-1} e^{x}$.

The new coordinate $x$ varies on the whole axis from $-\infty$ to $\infty$ with $x \rightarrow-\infty$ corresponding to the point $r=0$. By making the substitution

$w_{l}(\kappa ; r)=e^{x / 2} y_{l}(x)$ 
we transform Eq. (14) into

$y_{l}^{\prime \prime}(x)+q^{2}(x) y_{l}(x)=0$,

with

$q^{2}(x)=e^{2 x}\left(1-\frac{v(x)}{\kappa^{2}}\right)-\left(l+\frac{1}{2}\right)^{2}$.

Equation (25) can be solved already in the WKB approximation (see also [33]). The inverse transformation with respect to the coordinate $r$ gives us

$q^{2}(x)=r^{2} k_{l}^{2}(r)$,

where $k_{l}(r)$ is the quasiclassical wave number

$k_{l}(r)=\sqrt{\kappa^{2}-v_{\text {eff }}(r)}$,

expressed in terms of the reduced effective potential

$v_{\text {eff }}(r)=\frac{2 \mu}{\hbar^{2}} V_{\text {eff }}(r)$

with

$V_{\mathrm{eff}}(r)=V(r)+\frac{\hbar^{2}(l+1 / 2)^{2}}{2 \mu r^{2}}$.

The classical turning points $x_{1}, x_{2}$ and $x_{3}$ on the axis $x$ are the roots of the equation $q(x)=0$. They are related, respectively, to the turning points $r_{1}, r_{2}$, and $r_{3}$, where $k_{l}\left(r_{i}\right)=0$ (see Fig. 1).

Under the centrifugal barrier at $x<x_{1}$, the regular WKB solution is represented by the attenuating exponent:

$y_{l}(x)=\frac{C_{l}}{\sqrt{|q(x)|}} \exp \left(-\int_{x}^{x_{1}}\left|q\left(x^{\prime}\right)\right| d x^{\prime}\right)$.

By the conventional procedure [39], one finds the function in the potential well, where $x_{1}<x<x_{2}$,

$y_{l}(x)=\frac{2 C_{l}}{\sqrt{q(x)}} \cos \left(\int_{x_{1}}^{x} q\left(x^{\prime}\right) d x^{\prime}-\frac{\pi}{4}\right)$,

as well as under the Coulomb barrier, where $x_{2}<$ $<x<x_{3}$

$y_{l}(x)=\frac{C_{l}}{\sqrt{|q(x)|}}\left[\cos \alpha_{l} e^{-S_{l}(Q)} \exp \left(\int_{x}^{x_{3}}\left|q\left(x^{\prime}\right)\right| d x^{\prime}\right)-\right.$

$\left.-2 \sin \alpha_{l} e^{S_{l}(Q)} \exp \left(-\int_{x}^{x_{3}}\left|q\left(x^{\prime}\right)\right| d x^{\prime}\right)\right]$.

ISSN 2071-0194. Ukr. J. Phys. 2021. Vol. 66, No. 5

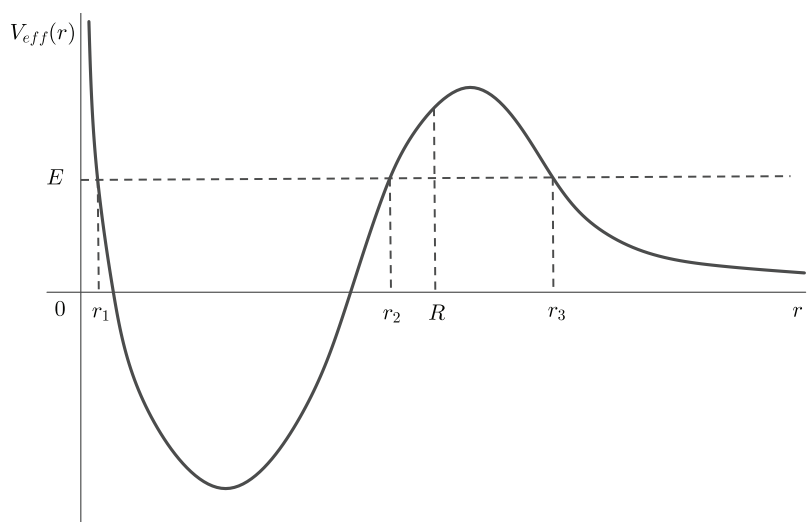

Fig. 1. Effective potential energy for the relative motion of the $\alpha$-particle and daughter nucleus, which incorporates the nuclear potential well, screened Coulomb interaction, and Lange's centrifugal barrier. $E=Q-\Delta Q$ is the mean kinetic energy of relative motion as $r \rightarrow \infty, Q$ is the mean nuclear energy released during the $\alpha$-decay, $\Delta Q$ is the energy absorbed by electrons. The classical turning points are denoted as $r_{1}, r_{2}$, and $r_{3}$. In the case of square nuclear potential well, the nuclear radius $R$ coincides with $r_{2}$

Here, the action

$S_{l}(Q)=\int_{x_{2}}^{x_{3}}\left|q\left(x^{\prime}\right)\right| d x^{\prime}$

and the angle

$\alpha_{l}=\int_{x_{1}}^{x_{2}} q(x) d x-\frac{\pi}{2}$

were introduced.

Behind the barrier for $x>x_{3}$,

$y_{l}(x)=-\frac{C_{l}}{\sqrt{q(x)}}\left[\cos \alpha e^{-S_{l}} \sin \left(\int_{x_{3}}^{x} q\left(x^{\prime}\right) d x^{\prime}-\frac{\pi}{4}\right)+\right.$

$\left.+4 \sin \alpha e^{S_{l}} \cos \left(\int_{x_{3}}^{x} q\left(x^{\prime}\right) d x^{\prime}-\frac{\pi}{4}\right)\right]$.

Let us return now to the radial coordinate $r$ by means of Eqs. (23), (24). Action (34) and angle (35) can be rewritten then as

$S_{l}(Q)=\int_{r_{2}}^{r_{3}}\left|k_{l}(r)\right| d r$ 
and

$\alpha_{l}=\int_{r_{1}}^{r_{2}} k_{l}(r) d r-\frac{\pi}{2}$

with $k_{l}(r)$ defined by Eq. (28). Equation (37) completely defines the barrier penetrability $e^{-2 S_{l}(Q)}$. It can be rewritten in a more familiar form:

$e^{-2 S_{l}(Q)}=\exp \left\{-\frac{2}{\hbar} \int_{r_{2}}^{r_{3}} \sqrt{2 \mu\left(V_{\mathrm{eff}}(r)-E_{d}\right)} d r\right\}$.

Starting from expression (36), one can represent the wave function behind the Coulomb barrier $(r>$ $\left.>r_{3}\right)$ in the form

$w_{l}(\kappa ; r)=C_{l}\left(\frac{\kappa}{k_{l}(r)}\right)^{1 / 2} X_{l} \sin \left(\int_{r_{3}}^{r} k_{l}(r) d r+\gamma+\frac{\pi}{4}\right)$,

where the new parameters $X_{l}$ and $\gamma$ satisfy the equations

$X_{l} \sin \gamma=\cos \alpha_{l} e^{-S_{l}}, \quad X_{l} \cos \gamma=-4 \sin \alpha_{l} e^{S_{l}}$,

whose solutions are

$X_{l}=\left[16 \sin ^{2} \alpha_{l} e^{2 S_{l}}+\cos ^{2} \alpha_{l} e^{-2 S_{l}}\right]^{1 / 2}$

and

$\gamma=-\arctan \left(\frac{e^{-2 S_{l}}}{4 \tan \alpha_{l}}\right)$.

At large distances, $k_{l}(r)$ approaches $\kappa$. Therefore, the integral in Eq. (40) can be rewritten as

$\int_{r_{3}}^{r} k_{l}(r) d r=\int_{r_{3}}^{\infty}\left[k_{l}(r)-\kappa\right] d r+\kappa\left(r-r_{3}\right)$.

Afterward, equating the function $w_{l}(\kappa ; r)$ with $r \rightarrow$ $\rightarrow \infty$ to its asymptotic form (17), one gets the amplitude

$C_{l}=\sqrt{\frac{2}{\pi}} \frac{1}{X_{l}}$

and the scattering phase shift

$\delta_{l}=\delta_{l}^{(0)}+\gamma$,

384 where $\gamma$ is given by (43) and

$\delta_{l}^{(0)}=\int_{r_{3}}^{\infty}\left[k_{l}(r)-\kappa\right] d r-\kappa r_{3}+\left(l+\frac{1}{2}\right) \frac{\pi}{2}$.

The amplitude squared $C_{l}^{2}$ determines both the rate of reactions with $\alpha$ projectiles and the $\alpha$-decay rate. Therefore, it is interesting to examine its behavior as a function of the deviation from the resonance $\delta \alpha_{l}=\alpha_{l}-n \pi$, where $n=1,2, \ldots$. Recall that the tunneling probability through the Coulomb barrier $e^{-2 S_{l}} \ll 1$. If $\left|\delta \alpha_{l}\right| \gg e^{-2 S_{l}} / 4$, the angle $\gamma$ and the amplitude are very small:

$\gamma=-\frac{1}{4} e^{-2 S_{l}} \cot \alpha_{l}, \quad C_{l}^{2}=\frac{1}{8 \pi} \frac{e^{-2 S_{l}}}{\sin ^{2} \alpha_{l}}$.

In the opposite case where $\left|\delta \alpha_{l}\right| \ll 1, C_{l}^{2}$ versus $\delta \alpha_{l}$ is described by the Lorentzian function

$C_{l}^{2}=C_{l, \mathrm{res}}^{2} \frac{\left(e^{-2 S_{l}} / 4\right)^{2}}{\left(\delta \alpha_{l}\right)^{2}+\left(e^{-2 S_{l}} / 4\right)^{2}}$,

where $C_{l, \text { res }}^{2}=2 e^{2 S_{l}} / \pi$ is the resonant value of $C_{l}^{2}$. This Lorentzian function can be rewritten as a function of the energy:

$C_{l}^{2}(E)=C_{l, \mathrm{res}}^{2} \frac{\left(\Gamma_{r} / 2\right)^{2}}{\left(E-E_{r}\right)^{2}+\left(\Gamma_{r} / 2\right)^{2}}$,

where $E_{r}$ is the resonant energy, corresponding to $\alpha_{l}=n \pi$, and $\Gamma_{r}$ the width of the resonance,

$\Gamma_{r}=\frac{e^{-2 S_{l}}}{2\left(\frac{d \alpha_{l}}{d E}\right)_{E=E_{r}}}$.

Thus, curve (50) has an extremely narrow width $\Gamma_{r} \sim e^{-2 S_{l}}$. In the same case, $\gamma$ is determined by the formula

$\gamma=-\arctan \left(\frac{e^{-2 S_{l}}}{4 \delta \alpha_{l}}\right)$,

which is easily reduced to the well-known expression [27]:

$\delta_{l}=\delta_{l}^{(0)}-\arctan \left(\frac{\Gamma_{r} / 2}{E-E_{r}}\right)$.

From here, we see that $\delta_{l}^{(0)}$ means that the phase shift is far from the resonance.

ISSN 2071-0194. Ukr. J. Phys. 2021. Vol. 66, No. 5 
According to (38), the resonant condition is satisfied, if

$\int_{r_{1}}^{r_{2}} k_{l}(r) d r=\left(n+\frac{1}{2}\right) \pi, \quad n=1,2, \ldots$.

This equality can be rewritten in the form

$\oint p_{l}(r) d r=2 \pi \hbar\left(n+\frac{1}{2}\right), \quad p_{l}=\hbar k_{l}$,

differing from the Bohr-Sommerfeld quantization rule [39] only by the extra term $\pi \hbar$. Equations (54) and (55) determine the energies of the quasistationary levels inside the potential well.

Similarly, by using (23), (31) and (33), one finds the WKB wave function inside the nucleus at $r_{1}<r<r_{2}$ :

$w_{l}(\kappa ; r)=2 C_{l} \sqrt{\frac{\kappa}{k(r)}} \cos \left(\int_{r_{1}}^{r} k_{l}\left(r^{\prime}\right) d r^{\prime}-\frac{\pi}{4}\right)$

and under the centrifugal barrier as $0<r<r_{1}$

$w_{l}(\kappa ; r)=C_{l} \sqrt{\frac{\kappa}{k_{l}(r)}} \exp \left(-\int_{r}^{r_{1}}\left|k_{l}\left(r^{\prime}\right)\right| d r^{\prime}\right)$.

For different nuclei, the deviation $\delta \alpha_{l}=\alpha_{l}-n \pi$, determined by the nuclear energy, takes arbitrary values in the interval ranging from $-\pi / 2$ to $\pi / 2$. Once the factor $e^{-2 S_{l}}$ is small, the probability for $\delta \alpha_{l}$ to occur in the narrow resonant interval $\sim e^{-2 S_{l}}$ will be extremely low. Therefore, below for the amplitude $C_{l}$, I use Eq. (48). Then, under the Coulomb barrier, $w_{l}(\kappa ; r)$ exponentially grows as $r$ increases:

$w_{l}(\kappa ; r)=\sqrt{\frac{2 \kappa}{\pi\left|k_{l}(r)\right|}} \exp \left(-\int_{r}^{r_{3}}\left|k_{l}\left(r^{\prime}\right)\right| d r^{\prime}\right)$,

where $r_{2}<r<r_{3}$. Behind the barrier, $r>r_{3}$, it is given by

$w_{l}(\kappa ; r)=\sqrt{\frac{2 \kappa}{\pi k_{l}(r)}} \sin \left(\int_{r_{3}}^{r} k_{l}\left(r^{\prime}\right) d r^{\prime}+\frac{\pi}{4}\right)$.

The irregular WKB solution $\tilde{w}_{l}(\kappa ; r)$ of the Schrödinger equation (14) is calculated in the same manner. It diverges at $r \rightarrow 0$,

$\tilde{w}_{l}(\kappa ; r) \sim \exp \left(\int_{r}^{r_{1}}\left|k_{l}\left(r^{\prime}\right)\right| d r^{\prime}\right)$, and has the asymptotics

$\tilde{w}_{l}(\kappa ; r) \approx-\sqrt{\frac{2}{\pi}} \cos \left(\kappa r-\frac{l \pi}{2}+\delta_{l}(\kappa)\right)$

at $r \rightarrow \infty$. Note that the phase shift $\delta_{l}(\kappa)$ is the same here as in the regular solution $w_{l}(\kappa ; r)$.

Again assuming that $e^{-2 S_{l}} \ll 1$, one finds that, in the region $r>r_{3}$,

$\tilde{w}_{l}(\kappa ; r)=-\sqrt{\frac{2 \kappa}{\pi k_{l}(r)}} \cos \left(\int_{r_{3}}^{r} k_{l}\left(r^{\prime}\right) d r^{\prime}+\frac{\pi}{4}\right)$,

while, under the Coulomb barrier,

$\tilde{w}_{l}(\kappa ; r)=-\sqrt{\frac{2 \kappa}{\pi\left|k_{l}(r)\right|}} \exp \left(\int_{r}^{r_{3}}\left|k_{l}\left(r^{\prime}\right)\right| d r^{\prime}\right)$

and, inside the nuclear potential well at $r_{1}<r<r_{2}$,

$\tilde{w}_{l}(\kappa ; r)=-\sqrt{\frac{2 \kappa}{\pi k_{l}(r)}} e^{S_{l}} \cos \left(\int_{r}^{r_{2}} k_{l}\left(r^{\prime}\right) d r^{\prime}-\frac{\pi}{4}\right)$.

In addition, let us introduce the complex functions

$d_{l}^{( \pm)}(\kappa ; r)=w_{l}(\kappa ; r) \pm i \tilde{w}_{l}(\kappa ; r)$,

which are described at $r \rightarrow \infty$ by the running waves:

$d_{l}^{( \pm)}(\kappa ; r) \approx \mp i \sqrt{\frac{2}{\pi}} \exp \left\{ \pm i\left[\kappa r-\frac{l \pi}{2}+\delta_{l}(\kappa)\right]\right\}$

Equation (14) is invariant with respect to the reflection of $\kappa$ to $-\kappa$. Therefore, its solutions $w_{l}(\kappa)$ and $\tilde{w}_{l}(\kappa)$ can only change the sign at such a transformation. Then in correspondence with their boundary conditions (17) and (61), one gets the following symmetry conditions:

$w_{l}(-\kappa ; r)=(-1)^{l+1} w_{l}(\kappa ; r)$,
$\tilde{w}_{l}(-\kappa ; r)=(-1)^{l} \tilde{w}_{l}(\kappa ; r)$.

The substitution of (67) into (65) gives another useful relation

$d_{l}^{(+)}(-\kappa ; r)=(-1)^{l} d_{l}^{(-)}(\kappa ; r)$.

In close vicinities to the turning points, the solutions are described by the Airy functions $A i(z)$ and 
$B i(z)[39,49]$. In particular, near the point $r_{3}$, the regular functions take the form

$w_{l}(\kappa ; r)=\sqrt{\frac{2 \kappa}{\left|k_{l}(r)\right|}}|z|^{1 / 4} A i(|z|) \quad$ at $\quad r \leq b$

and

$w_{l}(\kappa ; r)=\sqrt{\frac{2 \kappa}{k_{l}(r)}} z^{1 / 4} A i(-z)$ at $r \geq b$,

while the irregular functions become

$\tilde{w}_{l}(\kappa ; r)=-\sqrt{\frac{2 \kappa}{\left|k_{l}(r)\right|}}|z|^{1 / 4} B i(|z|), \quad$ at $\quad r \leq b$,

and

$\tilde{w}_{l}(\kappa ; r)=-\sqrt{\frac{2 \kappa}{k_{l}(r)}} z^{1 / 4} B i(-z), \quad$ at $\quad r \geq b$,

where

$z=\left(\frac{3}{2} \xi\right)^{2 / 3}$

with

$\xi(r)=\int_{b}^{r} k_{l}\left(r^{\prime}\right) d r^{\prime}$

\section{Evolution of the Wave}

\section{Packet which Describes the Alpha Decay}

Let the initial state $\Psi_{a}(0)=\varphi_{a}$ of the parent nucleus be formed at $t=0$. The time evolution of this wave function at $t \geq 0$ is governed by the equation [32]

$\Psi_{a}(t)=-\frac{1}{2 \pi i} \int_{-\infty}^{\infty} d \varepsilon e^{-i \varepsilon t / \hbar} \mathcal{G}^{+}(\varepsilon) \Psi_{a}(0)$,

where the retarded Green's operator

$\mathcal{G}^{+}(\varepsilon)=(\varepsilon+i \epsilon-H)^{-1}, \quad \epsilon \rightarrow+0$.

The wave function $\Psi_{a}(t)$ can be expanded in terms of the functions $\varphi_{a}$ and $\varphi_{b}^{+}$:

$\Psi_{a}(t)=c_{a}(t) \varphi_{a}+\sum_{b} c_{b}(t) \varphi_{b}^{+}$, where the sum over $b$ denotes the integral over the wave vector $\boldsymbol{\kappa}$, as well as the sum over quantum numbers of the daughter nucleus $I_{d} M_{d}$. The expansion coefficients are defined by

$c_{a(b)}(t)=-\frac{1}{2 \pi i} \int_{-\infty}^{\infty} d \varepsilon e^{-i \varepsilon t / \hbar}\left\langle\varphi_{a(b)}^{+}\left|\mathcal{G}^{+}(\varepsilon)\right| \varphi_{a}\right\rangle$,

where the Green matrix is determined by the relations $[32]$

$\mathcal{G}_{a a}^{+}(\varepsilon)=\frac{1}{\varepsilon-\varepsilon_{a}-\mathcal{R}_{a a}^{+}(\varepsilon)}$

and

$\mathcal{G}_{b a}^{+}(\varepsilon)=\frac{\mathcal{R}_{b a}^{+}(\varepsilon)}{\left(\varepsilon+i \epsilon-\varepsilon_{b}\right)\left(\varepsilon-\varepsilon_{a}-\mathcal{R}_{a a}^{+}(\varepsilon)\right)}$.

Here, $\mathcal{R}_{b a}^{+}(\varepsilon)=\mathcal{R}_{b a}(\varepsilon+i \epsilon)$ is the matrix of the level shift operator satisfying the integral equation [32]

$\mathcal{R}(\varepsilon)=V+V \frac{1-\mathcal{Q}_{a}}{\varepsilon-H_{0}} \mathcal{R}(\varepsilon)$

with the projection operator

$\mathcal{Q}_{a}=|a\rangle\langle a|$

on the initial state $|a\rangle$. The solution of Eq. (81) can be expanded in powers of $V$ :

$\mathcal{R}(\varepsilon)=V+V \frac{1-\Lambda_{a}}{\varepsilon-H_{0}} V+\ldots$.

The complex number $\mathcal{R}_{a a}^{+}\left(\varepsilon_{a}\right)$ is usually written down as [32]

$\mathcal{R}_{a a}^{+}\left(\varepsilon_{a}\right)=D\left(\varepsilon_{a}\right)-i \frac{\Gamma}{2}$,

where $D\left(\varepsilon_{a}\right)$ and $\Gamma$ mean the shift and width of the decaying parent level (below, for brevity, a small level shift $D\left(\varepsilon_{a}\right)$ will be omitted). The total width $\Gamma$ is a sum of all the partial widths:

$\Gamma=\sum_{b} \Gamma_{b}$

The partial $\alpha$ decay width reads

$\Gamma_{b}=2 \pi \sum_{M_{d}} \int d \Omega_{\hat{\boldsymbol{\kappa}}}\left|\mathcal{R}_{b a}^{+}\left(\varepsilon_{a}\right)\right|^{2} \varrho\left(\varepsilon_{b}\right)$,

ISSN 2071-0194. Ukr. J. Phys. 2021. Vol. 66, No. 5 
where the density of final states

$\varrho\left(\varepsilon_{b}\right)=\kappa_{b} \mu / \hbar^{2}$

depends on the wave number

$\kappa_{b}=\sqrt{2 \mu E_{b}} / \hbar$

The width (86) is proportional to the squared amplitude $C_{l}^{2}$ of the wave $\varphi_{b}^{+}$. Therefore, according to Eqs. (48) and (49), $\Gamma_{b} \sim e^{-2 S_{l}}$ far from the BohrSommerfeld condition, and $\Gamma_{b} \sim e^{2 S_{l}}$ in the case of resonance, as $\delta \alpha_{l}=0$. The latter corresponds to the immediate decay of the nucleus and contradicts all experimental data.

Green's functions (79), (80) have a pole at the point $\varepsilon_{0}=\mathcal{R}_{a a}^{+}\left(\varepsilon_{a}\right)$ on the second sheet of the complex Riemann $\varepsilon$ surface. Moreover, $\mathcal{G}_{b a}^{+}(\varepsilon)$ has a pole at $\varepsilon=\varepsilon_{b}-i \epsilon$. Inserting Green's functions into (78) and performing the contour integration (for details, see Ref. [32]), one arrives at

$\Psi_{a}(t)=\varphi_{a} e^{-i \varepsilon_{a} t / \hbar-\Gamma t / 2 \hbar}+$

$+\sum_{b} \frac{\mathcal{R}_{b a}^{+}\left(\varepsilon_{a}\right) \varphi_{b}^{+}}{\varepsilon_{b}-\varepsilon_{a}+i \frac{\Gamma}{2}}\left[e^{-i \varepsilon_{b} t / \hbar}-e^{-i \varepsilon_{a} t-\Gamma t / 2 \hbar}\right]$.

From here it immediately follows that the probability of finding the parent nucleus in the initial state is described by the exponential decay law [32]:

$P_{a}(t)=e^{-\Gamma t / \hbar}$.

The probability of finding the clusters at the moment $t$ with energy in the interval from $E$ to $E+\Delta E$ for the decay through the $d$ th channel can be written as

$\Delta P_{d}(E, t)=W_{d}(E, t) \Delta E$.

From Eq. (89), it follows that the probability density at $t \rightarrow \infty$ is

$W_{d}(E, \infty)=\frac{1}{\pi} \frac{\Gamma_{d} / 2}{\left(E-E_{d}\right)^{2}+(\Gamma / 2)^{2}}$.

The corresponding branching ratio will be

$\int_{0}^{\infty} W_{d}(E, \infty) d E=\frac{\Gamma_{d}}{\Gamma}$.

The level shift operator $\mathcal{R}(\varepsilon)$ is invariant with respect to rotations. Therefore, $\mathcal{R}(\varepsilon)$ only couples states ISSN 2071-0194. Ukr. J. Phys. 2021. Vol. 66, No. 5 with the same total angular momentum $I=I_{p}$ and its projection $M=M_{p}$. With (21), the off-diagonal matrix elements $\mathcal{R}_{b a}$ can be represented in the form

$\mathcal{R}_{b a}^{+}\left(\varepsilon_{a}\right)=\kappa^{-1} \sum_{l} \mathfrak{Y}_{I_{p}}^{M_{p}}\left(l I_{d} M_{d} ; \hat{\boldsymbol{\kappa}}\right) \mathscr{I}_{l I_{d}}(\kappa)$,

where $\mathscr{I}_{l I_{d}}(\kappa)$ denotes the integral

$\mathscr{I}_{l I_{d}}(\kappa)=\int d \mathbf{r}^{\prime} \frac{w_{l}\left(\kappa ; r^{\prime}\right)}{r^{\prime}} \times$

$\times \int d \xi \mathscr{Y}_{I_{p} l I_{d}}^{M_{p^{*}}}\left(\xi, \hat{\mathbf{r}}^{\prime}\right) \mathcal{R}^{+}\left(\varepsilon_{a}\right) g_{I_{p} M_{p}}\left(\xi, \mathbf{r}^{\prime}\right)$.

Next, substituting (94) into (86), one obtains the decay width $\Gamma_{b}$ :

$\Gamma_{b}=2 \pi \sum_{l}\left|\mathscr{I}_{l I_{d}}(\kappa)\right|^{2} \varrho\left(\varepsilon_{b}\right)$.

Here, $l$ runs the values from $\left|I_{d}-I_{p}\right|$ to $I_{d}+I_{p}$. The sum reduces to a single term, if $I_{p}$ or $I_{d}$ equals zero.

The relative motion of the clusters in the decay channel $d=I_{d} M_{d}$ is described by the wave function

$\Phi_{d}(\mathbf{r}, t)=\left\langle g_{d} g_{\alpha} \mid \Psi_{a}(t)\right\rangle e^{i\left(\varepsilon_{b}-E\right) t / \hbar}$.

With the aid of expression (89), one has

$\Phi_{d}(\mathbf{r}, t)=\frac{2 \mu}{\hbar^{2}} \int d \boldsymbol{\kappa} \frac{\mathcal{R}_{b a}^{+}\left(\varepsilon_{a}\right) \psi_{\kappa}^{+}(\mathbf{r})}{\kappa^{2}-\kappa_{d}^{2}+i \Gamma \mu / \hbar^{2}} \times$

$\times\left[e^{-i E t / \hbar}-e^{-i E_{d} t / \hbar-\Gamma t / 2 \hbar}\right]$.

If expressions (13) and (94) are inserted here, the calculation of the integral over the spherical angles $\hat{\boldsymbol{\kappa}}$ gives:

$$
\begin{aligned}
& \int d \Omega_{\kappa} \mathcal{R}_{b a}^{+}\left(\varepsilon_{a}\right) \psi_{\kappa}^{+}(\mathbf{r})= \\
& =\sum_{l m}\left(l I_{d} m M_{d} \mid I_{p} M_{p}\right) \mathscr{I}_{l I_{d}}(\kappa) \frac{w_{l}(\kappa ; r)}{\kappa^{2} r} Y_{l m}(\hat{\mathbf{r}}) .
\end{aligned}
$$

For calculation of the remaining integral over the radial variable $\kappa$, it is convenient to extend the integration to the whole region $-\infty<\kappa<\infty$. For this aim, the denominator of (98) is rewritten as

$\frac{1}{\kappa^{2}-\kappa_{d}^{2}+i \Gamma \mu / \hbar^{2}}=\frac{1}{2 \kappa_{0}}\left[\frac{1}{\kappa-\kappa_{0}}-\frac{1}{\kappa+\kappa_{0}}\right]$, 
where

$\kappa_{0} \approx \kappa_{d}-i \frac{\Gamma}{2 \hbar v_{d}}$,

$v_{d}=\hbar \kappa_{d} / \mu$ is the mean velocity of the relative motion of clusters.

Furthermore, it should be taken into account that $\mathscr{I}_{l I_{d}}(\kappa) w_{l}(\kappa ; r)$ is an even function of $\kappa$, and

$w_{l}(\kappa ; r)=\frac{d_{l}^{(+)}(\kappa ; r)+d_{l}^{(-)}(\kappa ; r)}{2}$,

Then expression (98) for the wave function transforms to

$\Phi_{I_{d} M_{d}}(\mathbf{r}, t)=\frac{\phi_{I_{d} M_{d}}(\mathbf{r}, t)}{r}$

with

$\phi_{I_{d} M_{d}}(\mathbf{r}, t)=-\pi i \frac{\mu}{\hbar^{2} \kappa_{d}} \sum_{l m}\left(l I_{d} m M_{d} \mid I_{p} M_{p}\right) \times$
$\times\left[\mathcal{F}_{l}^{+}(r, t)+\mathcal{F}_{l}^{-}(r, t)\right] Y_{l m}(\hat{\mathbf{r}})$,

where the following notations are introduced:

$\mathcal{F}_{l}^{ \pm}(r, t)=\frac{i}{2 \pi} \int_{-\infty}^{\infty} \frac{d_{l}^{ \pm}(\kappa ; r) \mathscr{I}_{l I_{d}}(\kappa)}{\kappa-\kappa_{0}} \times$

$\times\left[e^{-i E t / \hbar}-e^{-i E_{d} t / \hbar-\Gamma t / 2 \hbar}\right] d \kappa$.

Here, the integration is concentrated mainly around $\kappa_{d}$ in a very narrow interval $\Delta \kappa_{d}=\kappa-\kappa_{d}$ of the order of $\Gamma / \hbar v_{d}$. Therefore, it is possible to use the approximate equality

$e^{-i E t / \hbar} \approx e^{-i E_{d} t / \hbar} e^{-i v_{d} t \Delta \kappa_{d}}$.

Further, I will consider the wave function of the $\alpha$-decay only outside the nucleus, where the smooth function $\mathscr{I}_{l I_{d}}(\kappa)$ can be replaced by $\mathscr{I}_{l I_{d}}\left(\kappa_{d}\right)$. Then the function $\mathcal{F}_{l}^{ \pm}(r, t)$ transforms to

$\mathcal{F}_{l}^{ \pm}(r, t)=\mathscr{I}_{l I_{d}}\left(\kappa_{d}\right) I_{l}^{ \pm}(r, t) e^{-i E_{d} t / \hbar}$,

where

$I_{l}^{ \pm}(r, t)=\frac{i}{2 \pi} \int_{-\infty}^{\infty} \frac{d_{l}^{ \pm}(\kappa ; r)}{\kappa-\kappa_{0}} \times$
$\times\left[e^{-i v_{d} t\left(\kappa-\kappa_{d}\right)}-e^{-\Gamma t / 2 \hbar}\right] d \kappa$.

The integrand in (108) has a simple pole $\kappa_{0}=$ $=\kappa_{d}-i \Gamma / 2 \hbar v_{d}$ in the lower part of the complex $\kappa$ plane. If $v_{d} t<r$, the integration contour in $I_{l}^{+}(r, t)$ is supplemented by a semicircle $C$ of the radius $R \rightarrow \infty$ in the upper half-plane $\kappa=\kappa^{\prime}+i \kappa^{\prime \prime}$. If $v_{d} t>r$, it is done in the lower half-plane. The integration along these semicircles $C$, where $d_{l}^{ \pm}(\kappa, R) \sim e^{ \pm i \kappa R}$, gives zero. Then the integral $I_{l}^{+}$is easily calculated by means of the residue theory. As to the function $I_{l}^{-}(r, t)$, it appears to be proportional to the difference of two exponents $e^{-\Gamma t / 2 \hbar}$ at any time moment $t$. Hence, $I_{l}^{-}(r, t)=0$.

Finally the wave function outside the nucleus takes the form

$\phi_{I_{d} M_{d}}(\mathbf{r}, t)=-i \sqrt{\frac{\pi}{2}} \sum_{l m} \mathcal{A}_{I_{d} M_{d}}^{(l m)} d_{l}^{(+)}\left(\kappa_{0} ; r\right) Y_{l m}(\hat{\mathbf{r}}) \times$

$\times e^{-i E_{d} t / \hbar-\Gamma t / 2 \hbar} \Theta\left(t-r / v_{d}\right)$,

where the amplitude

$\mathcal{A}_{I_{d} M_{d}}^{(l m)}=-\sqrt{2 \pi} \frac{\mu}{\hbar^{2} \kappa_{d}}\left(l I_{d} m M_{d} \mid I_{p} M_{p}\right) \mathscr{I}_{l I_{d}}\left(\kappa_{d}\right)$

and the Heaviside step function

$\Theta(x)= \begin{cases}1, & x>0 \\ 0, & x<0\end{cases}$

Note that $d_{l}\left(\kappa_{0} ; r\right)$ in (109) depends on the complex number $\kappa_{0}$ defined by Eq. (101). Therefore, in the asymptotic region, where $d_{l}^{+}\left(\kappa_{0} ; r\right)$ is defined by Eq. (66), the wave function takes the form

$\phi_{I_{d} M_{d}}(\mathbf{r}, t)=\sum_{l m} \mathcal{A}_{I_{d} M_{d}}^{(l m)} e^{i\left(\kappa_{d} r-l \pi / 2+\delta_{l}\right)} Y_{l m}(\hat{\mathbf{r}}) \times$

$\times \exp \left[-i E_{d} t / \hbar-\Gamma\left(t-r / v_{d}\right) / 2 \hbar\right] \Theta\left(t-r / v_{d}\right)$.

We see that the emitted $\alpha$-particles are described by a spherically diverging wave, which propagates with the wave vector $\kappa_{d}$ and has a sharp wave front defined by the radial coordinate $r_{f}=v_{d} t$. The intensity of this wave $\mathcal{I}_{d}(r, t)=\left|\phi_{I_{d} M_{d}}(r, t)\right|^{2}$ in units of $\left|\phi_{I_{d} M_{d}}\left(r_{f}, t\right)\right|^{2}$ can be written as

$\mathcal{I}_{d}(r, t)=\exp \left[-\frac{t}{\tau_{n}}\left(1-\frac{r}{r_{f}}\right)\right]$,

where $\tau_{n}=\hbar / \Gamma$ is the nuclear lifetime. The dependence of the intensity $\mathcal{I}_{d}(r, t)$ on $r$ at the fixed time moment $t=2 \tau_{n}$ is shown in Fig. 2 .

The probability of that, at the time moment $t$, one can detect the $\alpha$-particle emitted in the $d$ th channel reads

$P_{d}(t)=\sum_{M_{d}} \int\left|\Phi_{I_{d} M_{d}}(\mathbf{r}, t)\right|^{2} d \mathbf{r}$. 
For large enough times, when $t \gg r_{a} / v_{d}$, the main contribution into this integral is from the region outside the atom of the radius $r_{a}$. Then, substituting the asymptotic expression (112) into (114), one immediately gets

$P_{d}(t)=\frac{\Gamma_{d}}{\Gamma}\left(1-e^{-\Gamma t / \hbar}\right)$.

According to Eq. (85), the sum of the decay probabilities into all possible channels and the survival probability (90) at any moment $t$ equals unity, which confirms the correctness of the above calculations.

\section{Moshinsky Function}

In the asymptotic region, one can avoid approximation (106) and calculate the wave function with the aid of the Moshinsky function [42-48]

$M\left(r, \kappa_{0}, t\right)=\frac{i}{2 \pi} \int_{-\infty}^{\infty} \frac{e^{-i \hbar \kappa^{2} t / 2 \mu} e^{i \kappa r}}{\kappa-\kappa_{0}} d \kappa$,

where $\operatorname{Im} \kappa_{0}<0$. Then the strict expression for the wave function $\phi_{I_{d} M_{d}}(\mathbf{r}, t)$ reads

$\phi_{I_{d} M_{d}}(\mathbf{r}, t)=\sum_{l m} \mathcal{A}_{I_{d} M_{d}}^{(l m)} M\left(r, \kappa_{0}, t\right) \times$

$\times e^{-i l \pi / 2+i \delta_{l}(\kappa)} Y_{l m}(\hat{\mathbf{r}})$.

The alternative expression for the Moshinsky function is [45]

$M\left(r, \kappa_{0}, t\right)=\frac{1}{2} e^{-i \hbar \kappa_{0}^{2} t / 2 \mu} e^{i \kappa_{0} r} \operatorname{erfc}(y)$,

where $\operatorname{erfc}(y)$ is the complementary error function [49]:

$\operatorname{erfc}(y)=1-\operatorname{erf}(y)$

expressed in terms of the error function

$\operatorname{erf}(y)=\frac{2}{\sqrt{\pi}} \int_{0}^{y} e^{-u^{2}} d u$

depending on

$y=e^{-i \pi / 4} x, \quad x=\left(\frac{\mu}{2 \hbar t}\right)^{1 / 2}\left(r-v_{0} t\right)$

and $v_{0}=\hbar \kappa_{0} / \mu$. Since $|\arg y|<\pi / 4$, one can choose the integration path in (120) as a direct line on the ISSN 2071-0194. Ukr. J. Phys. 2021. Vol. 66, No. 5

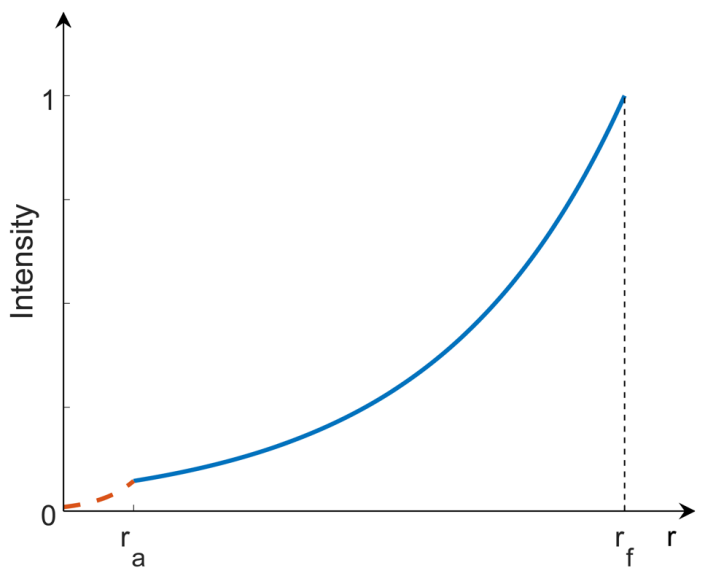

Fig. 2. Intensity dependence of the wave function, which describes the relative motion of the decay fragments, on the relative coordinate $r$ at $t=2 \tau_{n}$. The coordinate $r_{f}=v_{d} t$ determines the position of the wave front, and $r_{a}$ is the atom radius

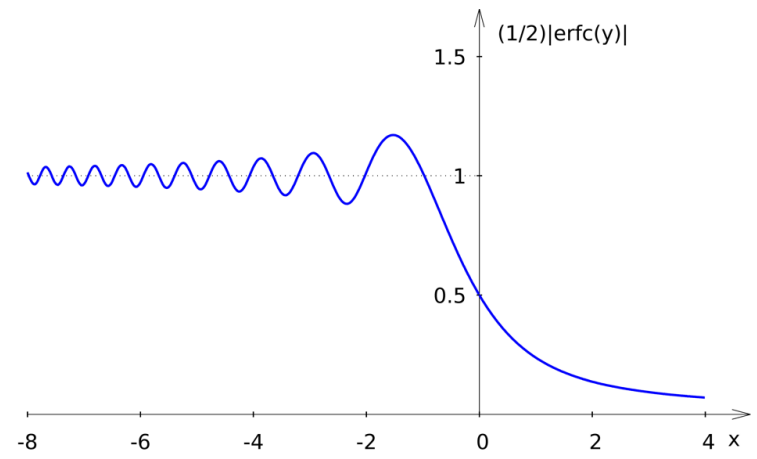

Fig. 3. Dependence of the function $(1 / 2)|\operatorname{erfc}(y)|$ with $y=$ $=e^{-i \pi / 4} x$ on the variable $x$

complex plane $u=u^{\prime}+i u^{\prime \prime}$, crossing the points $u=0$ and $y$.

Bearing in mind Eq. (101), one can reduce function (118) to

$M\left(r, \kappa_{0}, t\right)=e^{i \kappa_{d} r-i E_{d} t / \hbar} e^{-\Gamma\left(t-r / v_{d}\right) / 2 \hbar} \frac{1}{2} \operatorname{erfc}(y)$.

Here, $v_{0}$ is changed by $v_{d}$, since $\Gamma \ll E_{d}$. At last, making substitution (122) into (117), one arrives at the same expression (109) for the $\alpha$-wave function, but with $\Theta\left(t-r / v_{d}\right)$ replaced by $(1 / 2) \operatorname{erfc}(y)$.

At the wave front $r=r_{f}=v_{d} t$, the complementary error function $\operatorname{erfc}(0)$ equals unity by definition. For the analysis of its behavior at large values of $|y|$, let 
us make the substitution $u=e^{-i \pi / 4} \varsigma$ giving

$\operatorname{erf}(y)=\sqrt{\frac{2}{\pi}}(1-i)\left(\int_{0}^{x} \cos \varsigma^{2} d \varsigma+i \int_{0}^{x} \sin \varsigma^{2} d \varsigma\right)$.

As $|x| \rightarrow \infty$, these integrals reduce to the table integrals [50]

$\int_{0}^{\infty} \cos \varsigma^{2} d \varsigma=\int_{0}^{\infty} \sin \varsigma^{2} d \varsigma=\frac{1}{2} \sqrt{\frac{\pi}{2}}$

leading us to the conclusion that

$\frac{1}{2} \operatorname{erfc}(y) \rightarrow \begin{cases}1, & x \rightarrow-\infty \\ 0, & x \rightarrow \infty\end{cases}$

Thus, far from the point $y=0$, the $(1 / 2) \operatorname{erfc}(y)$ coincides with the Heaviside step function. The results of numerical calculations of $(1 / 2)|\operatorname{erfc}(y)|$ are displayed in Fig. 3. They demonstrate that the wave front is considerably distorted only in the interval $|x| \sim 10$.

It corresponds to the time interval $\Delta t=t-t_{0}$ of the order of

$|\Delta t| \sim \frac{10}{v_{d}} \sqrt{\frac{2 r}{\kappa_{d}}}$

where $t_{0}=r / v_{d}$ designates the arrival moment of the $\alpha$-wave packet to the detector located at the point $r$. Simple estimations show that, for the distance between the target and detector $\sim 1 \mathrm{~m}$, the energy $E_{d} \sim 5 \mathrm{MeV}$, the arrival time will be $t_{0} \sim 10^{-6} \mathrm{~s}$, and the time interval $|\Delta t| \sim 10^{-14}$ s. Unfortunately, such negligible time window makes the observation of the Moshinsky transient effect in the $\alpha$-decay to be unreal.

\section{Approximate Calculations}

Let us consider now the decay of even-even nuclei from the ground state, in which $I_{p}=0$ and $l=I_{d}$. Then the sum over $l$ in Eq. (96) reduces to the single term

$\lambda_{l} \sim\left|\mathscr{I}_{l}\left(\kappa_{l}\right)\right|^{2}$,

where the integral $\mathscr{I}_{l I_{d}}(\kappa)$ is defined by Eq. (95). It is natural to demand that the parameter $\left|\mathscr{I}_{l}\right|^{2}$ be determined by the overall intensity of the $\alpha$-wave inside the nucleus, i.e.,

$$
\left|\mathscr{I}_{l}\right|^{2}=\mathscr{N}^{2} \int_{r_{1}}^{r_{2}} \frac{w_{l}^{2}\left(\kappa_{l} ; r\right)}{\kappa_{l}^{2}} d r
$$

where the coefficient $\mathscr{N}$ has the dimensionality of energy. Writing (128), I neglected the exponentially attenuating tail of $w_{l}(\kappa ; r)$ under the centrifugal barrier. Then, far from the Bohr-Sommerfeld condition, the decay constant of the rigid nucleus transforms to

$$
\begin{aligned}
& \lambda_{l}=\mathscr{N}^{2} \frac{4 \mu}{\hbar^{3}} \frac{e^{-2 S_{l}}}{\sin ^{2} \alpha_{l}} \int_{r_{1}}^{r_{2}} \frac{d r}{k_{l}(r)} \times \\
& \times \cos ^{2}\left(\int_{r_{1}}^{r} k_{l}\left(r^{\prime}\right) d r^{\prime}-\frac{\pi}{4}\right) .
\end{aligned}
$$

As usual (see, e.g., Ref. [29]), the quickly oscillating squared cosine is replaced by $1 / 2$ giving

$T_{l}=\frac{2 \mu}{\hbar} \int_{r_{1}}^{r_{2}} \frac{d r}{k_{l}(r)}$

which may be interpreted as a quasiclassical period of the $\alpha$-particle oscillations inside the nucleus between the turning points $r_{1}$ and $r_{2}$. The corresponding knocking frequency is $\nu_{l}=1 / T_{l}$.

Then (129) is reduced to the expression

$\lambda_{l}=p_{l} \nu_{l} e^{-2 S_{l}}$

differing from the classical Gamov formula by the factor

$p_{l}=\frac{8 \pi^{2}}{\sin ^{2} \alpha_{l}}\left(\frac{\mathscr{N}}{\hbar \omega_{l}}\right)^{2}$,

which may be interpreted as a formation probability of the $\alpha$-particle. Here, $\hbar \omega_{l}$, where $\omega_{l}=2 \pi \nu_{l}$, means the phonon energy of the $\alpha$-particle vibrations. It is worth noting that Eq. (131) for $p_{l}=1$ coincides with the quasiclassical expression for $\lambda_{l}$ derived in [30].

Employing Eqs. (130)-(131) with $p_{l}=1$, I calculated the partial half-lives $T_{1 / 2}$ for the $\alpha$-decay of the ground states of even-even uranium isotopes into the rotational bands of thorium ones. The nuclear interaction is approximated by a square potential well with depth $U_{0}=55 \mathrm{MeV}$, whose radius is determined by the familiar expression (see, e.g., $[8,9]$ )

$R=r_{0}\left[(A-4)^{1 / 3}+4^{1 / 3}\right]$

ISSN 2071-0194. Ukr. J. Phys. 2021. Vol. 66, No. 5 
Partial half-lives (in seconds)

for the alpha-decay of the ground states of uranium isotopes into rotational bands of thorium $\left({ }_{92}^{A} \mathrm{U} \rightarrow{ }_{90}^{A-4} \mathrm{Th}\right)$

\begin{tabular}{|c|c|c|c|c|c|}
\hline$A$ & $I_{d}$ & $Q(\mathrm{MeV})$ & $T_{1 / 2}^{\exp }$ & $T_{1 / 2}^{\text {calc }}$ & $T_{1 / 2}^{\text {calc }}[12]$ \\
\hline \multirow[t]{4}{*}{230} & $0^{+}$ & 6.0308 & $2.67 \times 10^{6}$ & $2.85 \times 10^{6}$ & $2.48 \times 10^{6}$ \\
\hline & $2^{+}$ & 5.9586 & $5.62 \times 10^{6}$ & $1.04 \times 10^{7}$ & $6.51 \times 10^{6}$ \\
\hline & $4^{+}$ & 5.8044 & $4.73 \times 10^{8}$ & $1.93 \times 10^{8}$ & $5.53 \times 10^{7}$ \\
\hline & $6^{+}$ & 5.5835 & $2.57 \times 10^{12}$ & $1.67 \times 10^{10}$ & $1.43 \times 10^{9}$ \\
\hline \multirow[t]{4}{*}{232} & $0^{+}$ & 5.4513 & $3.19 \times 10^{9}$ & $3.34 \times 10^{9}$ & $3.39 \times 10^{9}$ \\
\hline & $2^{+}$ & 5.3935 & $6.89 \times 10^{9}$ & $1.17 \times 10^{10}$ & $8.49 \times 10^{9}$ \\
\hline & $4^{+}$ & 5.2645 & $7.25 \times 10^{11}$ & $2.08 \times 10^{11}$ & $7.00 \times 10^{10}$ \\
\hline & $6^{+}$ & 5.0731 & $4.26 \times 10^{15}$ & $1.88 \times 10^{13}$ & $1.89 \times 10^{12}$ \\
\hline \multirow[t]{3}{*}{234} & $0^{+}$ & 4.8954 & $1.09 \times 10^{13}$ & $0.99 \times 10^{13}$ & $1.00 \times 10^{13}$ \\
\hline & $2^{+}$ & 4.8422 & $2.73 \times 10^{13}$ & $3.71 \times 10^{13}$ & $2.70 \times 10^{13}$ \\
\hline & $4^{+}$ & 4.7213 & $3.87 \times 10^{15}$ & $8.19 \times 10^{14}$ & $2.74 \times 10^{14}$ \\
\hline \multirow[t]{4}{*}{236} & $0^{+}$ & 4.6109 & $9.99 \times 10^{14}$ & $1.00 \times 10^{15}$ & $1.08 \times 10^{15}$ \\
\hline & $2^{+}$ & 4.5614 & $2.84 \times 10^{15}$ & $3.85 \times 10^{15}$ & $2.97 \times 10^{15}$ \\
\hline & $4^{+}$ & 4.4486 & $4.93 \times 10^{17}$ & $8.96 \times 10^{16}$ & $3.17 \times 10^{16}$ \\
\hline & $6^{+}$ & 4.2775 & $5.28 \times 10^{20}$ & $1.30 \times 10^{19}$ & $1.38 \times 10^{18}$ \\
\hline \multirow[t]{3}{*}{238} & $0^{+}$ & 4.3078 & $1.78 \times 10^{17}$ & $2.35 \times 10^{17}$ & $4.10 \times 10^{17}$ \\
\hline & $2^{+}$ & 4.2582 & $6.71 \times 10^{17}$ & $9.96 \times 10^{17}$ & $1.26 \times 10^{18}$ \\
\hline & $4^{+}$ & 4.1448 & $1.81 \times 10^{20}$ & $2.98 \times 10^{19}$ & $1.77 \times 10^{19}$ \\
\hline
\end{tabular}

with $r_{0}=1.27 \mathrm{fm}$. The results are compared in Table with the experimental data and calculations made by Denisov and Khudenko [12]. It is curious that the square potential rather well reproduces the experiment. This success of the simple square potential is tightly bound to the fact, lying in the basis of the Bohr-Mottelson equations, that the density of nuclei is practically constant through the nuclear volume. Although I considered only spherical nuclei, my calculations agree with the experimental data for deformed nuclei at spin $I>0$ somewhat better than the results of Ref. [12]. This can be explained by the fact that these authors did not account for the dependence of the assault frequency $\nu_{l}$ on the orbital momentum $l$ and, moreover, took a standard centrifugal barrier $\sim l(l+1)$ instead of the quasiclassical one $\sim(l+1 / 2)^{2}$.

\section{Conclusion}

So, a straightforward solution of the time-dependent Schrödinger equation is reported for the $\alpha$-decay. It is based on the idea that the parent nucleus, obtained at $t=0$ in any nuclear process, occurs in the bound state $\varphi_{a}$ described by the shell model treating all the nucleons as an ideal gas. Each level in the potential well occupied by nucleons has a negative energy lying lower than the continuous spectrum. Therefore, the wave function $\varphi_{a}$ corresponds to a really bound state. Such a function is orthogonal to the scattering functions $\varphi_{b}^{+}$of the continuous spectrum, which describe, in turn, the $\alpha$-particle and the daughter nucleus. Only the residual interaction $V^{\prime}$ gives rise to the decay of the state $\varphi_{a}$, by coupling it with the states $\varphi_{b}^{+}$.

The value of the isolated energy level $\varepsilon_{a}$ of the initial state is dictated by the character of the nuclear forces, Pauli principle, etc., but not by our desire to fulfil the Bohr-Sommerfeld quantization rule (54). Recall that this requirement determines the resonance levels $E_{r}$ in the scattering of particles by any potential well [27]. At these energies the density of the wave function of the incident particle inside the well reaches a maximum, while the scattering crosssection attributes a bump with the width $\Gamma_{r}$. This is well illustrated for the scattering of $\alpha$-particles by numerical calculations [28]. Furthermore, if there is an $\alpha$-wave packet inside the nucleus at $t=0$, which is spread in the energy interval $\Delta E \gg \Gamma_{r}$ and is concentrated at the resonance energy $E_{r}$, then it exponentially decays later with the lifetime $\tau=\hbar / \Gamma_{r}[27]$. It is curious that the decay constant $\lambda=1 / \tau$ for such a quasistationary level derived in Eq. (51) is easily transformed to the Gamov formula $\lambda=\nu e^{-2 S}$ with $\nu$ being reciprocal to the period of vibrations $T$ determined by Eq. (130).

According to Eqs. 95) and (96), the $\alpha$-decay width $\Gamma$ is proportional to the squared amplitude of the wave function $C_{l}^{2}$. In accordance with Eqs. (48) and (50), the $C_{l}^{2} \sim e^{-2 S_{l}}$ far from the resonance, while $C_{l}^{2} \sim e^{2 S_{l}}$, if the resonance condition (54) is exactly fulfilled. Hence, only far from the resonance in nonvibrating spherical nuclei, we come to the correct result $\lambda \sim e^{-2 S}$. In the case of a decay deeply under the barrier, the probability of that the energy $\varepsilon_{a}$ of the parent nucleus is lying in the narrow resonance window $\Gamma \sim e^{-2 S_{l}}$, is too tiny to be realized in a rigid nucleus.

In standard approach to the problem, the stationary Schrödinger equation is solved, which resulted in the complex energy and in the exponentially diverging wave function, which is spread over the whole space. Instead, I have constructed the wave packet 
$\Phi(r, t)$ as a superposition of the scattering wave functions. These functions are calculated quasiclassically. Exponentially growing with $r$ outside the nucleus, the $\Phi(r, t)$ is truncated at the point $r_{f}=v_{d} t$, which ensures its proper normalization. The profound analysis has shown that some distortions of the wave front, calculated by means of strict Moshinsky's approach, appear in a very narrow space-time interval and, therefore, can be ignored in the experiment.

Equations (48) and (50) were transformed to the quasiclassical expression (131), which was used in numerical calculations of $T_{1 / 2}$ for the $\alpha$-decay of the uranium isotopes into the rotational bands of the daughter nuclei. Although the calculations agree with the experimental data somewhat better than the results in [12], there remains a large discrepancy of the calculations with experiment, as the spin $I_{d}$ of a daughter nuclei grows. For explanation of this effect, a dependence of the potential energy on the deformation parameter $\beta$ will be taken into consideration in the next paper.

1. D.M. Brink, M.C. Nemes, D. Vautherin. Effect of intrinsic degrees of freedom on the quantum tunneling of a collective variable. Ann. Phys. 147, 171 (1983).

2. R.G. Lovas, R.J. Liotta, A. Insoliac, K. Vargaa, D.S. Deliond. Microscopic theory of cluster radioactivity. Phys. Rep. 294, 265 (1998).

3. S.G. Kadmensky, S.D. Kurgalin, Yu.M. Chuvilskii. Cluster states in atomic nuclei and cluster-decay processes. Phys. J. Part. Nucl. 38, 1333 (2007).

4. A. Sandulescu, W. Greiner. Cluster decays. Rep. Prog. Phys. 55, 1423 (1992).

5. D.S. Delion. Theory of Particles and Clusters Emission (Springer, 2010) [ISBN 978-3-642-14406-6].

6. R.G. Thomas. A formulation of the theory of alphaparticle decay from time-independent equations. Prog. Theor. Phys. 12, 253 (1954)

7. H.F. Zhang, G. Royer. A particle preformation in heavy nuclei and penetration probability. Phys. Rev. C 77, 054318 (2008).

8. A. Zdeb, M. Warda, K. Pomorski. Half-lives for $\alpha$ and cluster radioactivity in a simple model. Phys. Scr. T 154, 014029 (2013).

9. A. Zdeb, M. Warda, K. Pomorski. Half-lives for $\alpha$ and cluster radioactivity within a Gamow-like model. Phys. Rev. C 87, 024308 (2013).

10. I. Silisteanu, W. Scheid, A. Sandulescu. Proton, alpha and cluster decay rates for nuclei with $52 \leq Z \leq 56$ and $52 \leq$ $\leq N \leq 60$. Nucl. Phys. A 679, 317 (2001).

11. Daming Deng, Zhongzhou Ren. Improved double-folding $\alpha$-nucleus potential by including nuclear medium effects. Phys. Rev. C 96, 064306 (2017).
12. V.Yu. Denisov, A.A. Khudenko. $\alpha$ decays to ground and excited states of heavy deformed nuclei. Phys. Rev. C 80, 034603 (2009).

13. V.Yu. Denisov, A.A. Khudenko. Erratum: $\alpha$ decays to ground and excited states of heavy deformed nuclei. Phys. Rev. C 80, 034603 (2009); Phys. Rev. C 82, 059902(E) (2010).

14. M. Ismail, A.Y. Ellithi, M.M. Botros, A. Abdurrahman. Penetration factor in deformed potentials: Application to $\alpha$ decay with deformed nuclei. Phys. Rev. C 86, 044317 (2012).

15. Chang $\mathrm{Xu}$, Zhongzhou Ren. Favored $\alpha$-decays of medium mass nuclei in density-dependent cluster model. Nucl. Phys. A 760, 303 (2005).

16. Chang $\mathrm{Xu}$, Zhongzhou Ren. New deformed model of $\alpha$ decay half-lives with a microscopic potential. Phys. Rev. C 73, 041301(R) (2006).

17. D.F. Jackson, M. Rhoades-Brown. Theories of alpha-decay. Ann. Phys. 105, 151 (1977).

18. T. Berggren, P. Olandes. Alpha decay from deformed nuclei:(I). Formalism and application to ground-state decays. Nucl. Phys. A 473, 189 (1987).

19. T. Berggren, P. Olandes. Alpha decay from deformed nuclei: (II). Application to the decay of high-spin states. Nucl. Phys. A 473, 221 (1987).

20. T. Berggren. Anisotropic alpha decay from oriented oddmass isotopes of some light actinides. Phys. Rev. C 50, 2494 (1994).

21. Xiao-Dong Sun, Ping Guo, Xiao-Hua Li. Systematic study of $\alpha$ decay half-lives for even-even nuclei within a twopotential approach. Phys. Rev. C 93, 034316 (2016).

22. Xiao-Dong Sun, Chao Duan, Jun-Gang Deng, Ping Guo, Xiao-Hua Li. Systematic study of $\alpha$ decay for odd- $A$ nuclei within a two-potential approach. Phys. Rev. C 95, 014319 (2017).

23. B. Sahu, S. Bhoi. Potential for $\alpha$-induced nuclear scattering, reaction and decay, and a resonance-pole-decay model with exact explicit analytical solutions. Phys. Rev. C 96, 044602 (2017).

24. R.M. Clark, H.L. Crawford, A.O. Macchiavelli, D. Rudolph, A. Semark-Roth, C.M. Campbell, M. Cromaz, P. Fallon, C. Morse, C. Santamaria. $\alpha$ decay of high-spin isomers in $N=84$ isotones. Phys. Rev. C 99, 024325 (2019).

25. G. Gamow. Zur Quantentheorie des Atomkernes. Z. Phys. 51, 204 (1928).

26. R.W. Gurney, E.U. Condon. Quantum mechanics and radioactive disintegration. Phys. Rev. 33, 127 (1929).

27. A.G. Sitenko. Lectures in Scattering Theory. Edited by P.J. Shepherd (Elsevier, 1971) [ISBN: 9781483486825].

28. F.F. Karpeshin, G. LaRana, E. Vardaci, A. Brondi, R. Moro, S.N. Abramovich, V.I. Serov. Resonances in alphanuclei interaction. J. Phys. G: Nucl. Part. Phys. 34, 587 (2007).

29. S.A. Gurvitz, G. Kalbermann. Decay width and the shift of a quasistationary state. Phys. Rev. Lett. 59, 262 (1987).

ISSN 2071-0194. Ukr. J. Phys. 2021. Vol. 66, No. 5 
30. S.A. Gurvitz. Novel approach to tunneling problems. Phys. Rev. A 38, 1747 (1988).

31. S.A. Gurvitz, P.B. Semmes, W. Nazarewicz, T. Vertse. Modified two-potential approach to tunneling problems. Phys. Rev. A 69, 042705 (2004).

32. M.L. Goldberger, K.M. Watson. Collision Theory, (J. Wiley, 1964) [ISBN: 978-0486435077].

33. A.Ya. Dzyublik. Integrable wave function, describing space-time evolution of alpha-decay. arXiv: 2001.09505v1[nucl-th]26Jan2020.

34. A.Ya. Dzyublik. Alpha decay of deformed even-even nuclei. Acta Phys. Polonica 10, 69 (2017).

35. M. Freer, M. Freer, H. Horiuchi, Y. Kanada-En'yo, D. Lee, Ulf-G. Meißner. Microscopic clustering in light nuclei. Rev. Mod. Phys. 90, 035004 (2018).

36. N.T. Zinner. Alpha decay rate enhancement in metals: An unlikely scenario. Nucl. Phys. A 781, 81 (2007).

37. A.Ya. Dzyublik. Influence of electronic environment on $\alpha$ decay. Phys. Rev. C 90, 054619 (2014).

38. R.G. Newton. Scattering Theory of Waves and Particles (Springer, 1982) [ISBN: 978-3-642-88128-2].

39. A.S. Davydov. Quantum Mechanics. Edited by D. ter Haar (Elsevier, 1965) [ISBN: 9781483187839].

40. G. Esposito. A phase-integral perspective on $\alpha$-decay. Eur. Phys. J. Plus 135, 692 (2020).

41. R.E. Langer. On the connection formulas and the solutions of the wave equation. Phys. Rev. 51, 669 (1937).

42. M. Moshinsky. Boundary conditions and time-dependent states. Phys. Rev. 84, 525 (1951).

43. M. Moshinsky. Diffraction in time. Phys. Rev. Lett. 88, 625 (1952)

44. R.G. Winter. Evolution of a quasi-stationary state. Phys. Rev. 123, 1503 (1961)
45. G. Garcia-Calderón, A. Rubio. Transient effects and delay time in the dynamics of resonant tunneling. Phys. Rev. A 55, 3361 (1997)

46. W. van Dijk, Y. Nogami. Novel expression for the wave function of a decaying quantum system. Phys. Rev. Lett. 83, 2867 (1999).

47. W. van Dijk, F. Kataoka, Y. Nogami. Space-time evolution of a decaying quantum state. J. Phys. A 32, 6347 (1999).

48. W. van Dijk. Numerical time-dependent solutions of the Schrödinger equation with piecewise continuous potentials. Phys. Rev. E 93, 063307 (2016).

49. Handbook of Mathematical Functions. Edited by M. Abramovitz, I.A. Stegun (Nat. Bureau of Standards, 1972).

50. I.S. Gradshteyn, I.M. Ryzhik. Table of Integrals, Series, and Products. Edited by D. Zwillinger (Academic Press, 2007) [ISBN: 978-0-12-373637-6].

Received 08.07.20

О.Я. Дзюблик

\section{ПОСЛІДОВНА ТЕОРІЯ АЛЬФА-РОЗПАДУ}

В рамках теорії розпадів Гольдбергера і Ватсона $\alpha$-розпад розглядається як перехід між початковим зв'язаним станом ядра та станами неперервного спектра з $\alpha$-частинкою. В квазикласичному наближенні виведені базисні хвильові функції для $\alpha$-розпаду з довільним орбітальним кутовим моментом. Альфа-частинка описується квадратичноінтегровним хвильовим пакетом, утвореним цими функціями, амплітуда якого ззовні ядра експоненційно росте аж до хвильового фронту. Аналізується ефект Мошинського пошкодження хвильового фронту. Виведений вираз для швидкості розпаду апроксимується квазикласичною формулою.

$K л$ ю и в $i$ слов в: теорія розпаду, $\alpha$-розпад, тунелювання, теорія розсіювання. 